\title{
GENDER DIFFERENCES IN THE SCANDINAVIAN CYSTIC FIBROSIS POPULATION
}

\begin{tabular}{|c|c|}
\hline Journal: & Pediatric Pulmonology \\
\hline Manuscript ID: & PPUL-09-0392.R1 \\
\hline Wiley - Manuscript type: & Original Article \\
\hline $\begin{array}{l}\text { Date Submitted by the } \\
\text { Author: }\end{array}$ & 14-Mar-2010 \\
\hline Complete List of Authors: & $\begin{array}{l}\text { Olesen, Hanne; Aarhus University Hospital Skejby, Pediatric dept. A } \\
\text { Pressler, Tacjana; Copenhagen University Hospital, Cystic fibrosis } \\
\text { Center, Department of Paediatrics } \\
\text { Hjelte, Lena; Karolinska Institutet, Stockholm CF Center; Karolinska } \\
\text { Institutet, Pediatric } \\
\text { Mared, Lena; Lund University Hospital, Lund CF Center, Heart and } \\
\text { Lung Center } \\
\text { Lindblad, Anders; Queen Silvia Children's Hospital, Cystic Fibrosis } \\
\text { Centre } \\
\text { Knudsen, Per; Oslo University Hospital Ulleval, National Center for } \\
\text { Cystic Fibrosis } \\
\text { Laerum, Birger; Haukeland University Hospital, Department of } \\
\text { Thoracic Medicine } \\
\text { Johannesson, Marie; Uppsala University, Department of Public } \\
\text { Health and Caring Sciences; Family Medicine and Clinical } \\
\text { Epidemiology Section }\end{array}$ \\
\hline Keywords: & $\begin{array}{l}\text { cystic fibrosis, gender, pseudomonas, burkholderia, lung function, } \\
\text { steroid }\end{array}$ \\
\hline
\end{tabular}

\section{ScholarONE \\ Manuscript Central}




\section{GENDER DIFFERENCES IN THE SCANDINAVIAN CYSTIC FIBROSIS POPULATION}

1. Hanne Vebert Olesen; Department of Paediatrics, Aarhus University Hospital Skejby, DK-8200 Aarhus N, Denmark

2. Tacjana Pressler; Cystic fibrosis Center, Department of Paediatrics, Copenhagen University Hospital, Copenhagen, Denmark

3. Lena Hjelte; Stockholm CF Center, Karolinska University Hospital Huddinge, Karolinska Institutet, SE-141 86 Stockholm, Sweden

4. Lena Mared; Lund CF Center, Heart and Lung Center, Lund University Hospital, SE-221 85 Lund, Sweden

5. Anders Lindblad; Gothenburg Cystic Fibrosis Center, Sahlgrenska University Hospital, SE-416 85 Gothenburg, Sweden

6. Per Kristian Knudsen; National Center for Cystic Fibrosis, Oslo University Hospital Ulleval, N0407 Oslo, Norway.

7. Birger N. Lærum; Department of Thoracic Medicine, Haukeland University Hospital, N-5021 Bergen, Norway

8. Marie Johannesson; Department of Public Health and Caring Sciences; Family Medicine and Clinical Epidemiology Section, Uppsala University, 751 05, Uppsala, Sweden, and Department of Paediatrics and Child Health, University of Otago, ,Wellington Campus, Wellington, New Zealand

On behalf of the Scandinavian Cystic Fibrosis Study Consortium* 
*Scandinavian Cystic Fibrosis Study Consortium (additional members):

Norway:

Pål Leyell Finstad, National Center for Cystic Fibrosis, Oslo University Hospital Ullevaal, Oslo;

Gjermund Fluge, Department of Pediatrics, Haukeland University Hospital, Bergen;

Helge Michalsen, Pediatric Department, Sørlandet Hospital, Arendal;

Bjørn Skrede, National Center for Cystic Fibrosis, Oslo University Hospital Ullevaal, Oslo;

Olav-Trond Storrøsten, National Center for Cystic Fibrosis, Oslo University Hospital Ulleval, Oslo.

Nils Olav Hermansen, Department of Microbiology, Oslo University Hospital Ullevaal, Oslo.

Sweden:

Anne Geborek, Stockholm Cystic Fibrosis Centre, Karolinska University Hospital Huddinge, Stockholm;

Marita Gilljam, Dept Pulmonary Medicine and Allergology, Sahlgrenska University Hospital, Gothenburg;

Ferenc Karpati, Stockholm Cystic Fibrosis Centre, Karolinska University Hospital Huddinge, Karolinska

Institutet, Stockholm;

Mary Kämpe, Uppsala CF Center, Uppsala University Hospital, Uppsala,

Peter Meyer, Heart and Lung Center, Lund University Hospital, Lund;

Denmark:

Peter Oluf Schiøtz, Department of Pediatrics, Aarhus University Hospital Skejby, Aarhus;

Niels Høiby, Department of Clinical Microbiology, Copenhagen University Hospital, Copenhagen

\author{
Corresponding author: Hanne Vebert Olesen, Aarhus University Hospital Skejby, \\ Department of Pediatrics, Brendstrupgaardsvej 100, 8200 Aarhus N, Denmark. \\ E-mail: hvo@dadlnet.dk; Phone: +45 894967 40; fax: +45 89496023
}


Part of this study was presented as an oral presentation at the $29^{\text {th }}$ European Cystic Fibrosis

Conference, Copenhagen, June 15-18 2006

6
7

8

9

10

11

12

13

14

15

16

17

18

19

20

21 


\section{ABSTRACT}

\section{Aims.}

To explore whether gender differences in the Scandinavian CF patients exist in the areas of key clinical parameters, complications and medication.

Methods. Cross-sectional data on 890 (416 female) pancreatic insufficient CF patients were

evaluated regarding chronic infection, body mass index, lung function, medication, and diabetes, as well as data of pseudomonas infection status, antibiotic treatment and hospitalization from one year follow-up.

Results. We found no differences in lung function, body mass index, or frequency of diabetes. The adult group consisted of more males than females (208:168). We found no significant difference in prevalence of chronic Pseudomonas aeruginosa infection, but during the follow-up the incidence of new chronic infection was higher in adult females (10/33 vs. 4/56). Females had higher prevalence of Burkholderia infection (21/416 vs 11/474).

Adult females had more days on intravenous antibiotics (median 39 vs. 26 days/y), and days in hospital (median 2 vs 0 days/y). More adult females received inhaled and oral steroids. In the pediatric cohort, females were treated more often with macrolides as an anti-inflammatory agent.

Conclusion. We found no gender difference in key clinical parameters in our CF population. However, our study showed a higher risk of Pseudomonas and Burkholderia infection among the female patients. Additionally, we found that female patients require more intensified treatment regarding antibiotics, macrolides, steroids and days of hospitalization, indicating a true female disadvantage even with modern aggressive treatment. 


\section{Page 5 of 46}

Pediatric Pulmonology

The finding of more males than females in the adult population suggesting a male advantage, $\underline{\text { warrants a mortality study }} \rightarrow$

Deleted: However, more males than females in the adult population implied a gender difference in survival. We saw a gender difference including higher risk of Pseudomonas and Burkholderia infection in females, and more intensified

treatment in females regarding

antibiotics, macrolides as an

inflammatory agent, oral/inhaled steroids and days of hospitalization, indicating a

true female disadvantage even with

modern aggressive treatment. 


\section{BACKGROUND}

A gender gap is documented in the course of cystic fibrosis (CF) with a female disadvantage regarding morbidity and mortality ${ }^{1-3}$. Several factors have been suggested as contributing to this gap. In CF related diabetes (CFRD), both earlier debut and considerably poorer survival in females have been reported ${ }^{4 ; 5}$. Chronic infection with Pseudomonas aeruginosa occurs earlier in females ${ }^{6}$. While these factors all contribute to morbidity and mortality, they do not explain the underlying difference. A few studies have tried to evaluate this difference. Focus has been on psychosocial differences, as well as hormonal differences. Female patients have less energy intake than males (compared to daily recommended intake), perform less physical exercise, and express an overestimation of their own weight ${ }^{7-9}$. Variations in lung function during the menstrual cycle points to effects of female sex hormones ${ }^{10}$, recently supported by a study of the influence of estrogen on the function of alternative chloride channels ${ }^{11}$. Other authors have suggested the lack of anabolic effects of male sex hormones as a factor ${ }^{5}$. Hence, there is considerable evidence that a true gender gap exists in cystic fibrosis. However, a recent single-center study showed no gender gap when comparing key values like lung function, height and weight in children and adolescents ${ }^{12}$. The authors suggest that a closing of the gap is possible, even mandatory, with standardized intensive care.

\section{Working hypothesis:}

No gender differences exist among Scandinavian Cystic Fibrosis patients since by tradition we have a very proactive and easily accessible care for all our patients.

Aims:

To explore whether gender differences in the Scandinavian CF patients exist in the areas of key clinical parameters, complications and medication. 


\section{METHODS:}

\section{Study design:}

This study was part of a larger multi centre study on the prevalence and incidence of Pseudomonas aeruginosa (PA) infection in the Scandinavian CF cohort ${ }^{13}$. All eight CF centres in Scandinavia participated. Data were collected from 2001 to 2003. Overall, all Scandinavian countries have very high standards of living, and a government-financed health system with a high standard of centralised CF care. Some differences in $\mathrm{CF}$ care do exist however, such as a higher degree of shared care in northern Sweden and Norway due to geography, and somewhat different antibiotic and segregation policies. The differences between countries and centres have been presented in the main article within this project ${ }^{13}$. For the scope of this article, we focused only on the gender perspective. The treatment policies for male and female $\mathrm{CF}$ patients were similar within the individual center.

All CF patients, followed at the eight Scandinavian CF centres at the time of inclusion, were eligible for the study. Approximately $86 \%$ of all patients participated, allowing for a $>90 \%$ participation of patients eligible for the study (= non-transplanted). Patients were recruited consecutively at routine visits to the centres, when in a clinically stable condition. Exclusion criterion was lung transplant prior to inclusion, since this was likely to change the infection status, lung function and body mass index of the patients. CF was diagnosed as either two known CFcausing mutations in the $\mathrm{CF}$ transmembrane conductance regulator (CFTR) gene, and/or two positive sweat-tests together with symptoms compatible with the disease. Data recorded at inclusion were: age, height, weight, $\mathrm{BMI}$, lung function $\left(\mathrm{FEV}_{1}\right)$, chronic infections (based on regular cultures, 
as well as antibodies against PA, see below), regular medication at time of inclusion, pancreatic insufficiency (PI), CF related diabetes (CFRD) and meconium ileus (MI). Patients using pancreatic enzymes were classified as PI. Diabetes was defined as CFRD with fasting hyperglycemia using the WHO criteria ${ }^{14}$. Continuous macrolide treatment was recorded exclusively for patients with this drug prescribed as an anti-inflammatory agent ${ }^{15}$. Other regular medication was recorded if prescribed at inclusion (oral and inhaled steroids, ursodeoxycholic acid, mucolytics, and insulin). The patients were followed for one year, recording number of days in hospital and number of days on inhaled or intravenous (i.v.) antibiotics. At the end of the study period new PA antibodies were measured and infection status was reported. Patients 19 years or older at inclusion were designated as adults. For calculation of BMI z-score the reference values of Nysom was used ${ }^{16}$. For patients $\geq 19$ years the reference values from European Coal and Steel Community were used for calculating $\mathrm{FEV}_{1}$ percent of predicted $^{17}$, and for those $<19$ years Solymar reference values were used ${ }^{18}$. Days on inhaled or intravenous antibiotics and hospitalisation during the study period were recorded and calculated as days/year. For patients who died or underwent lung transplantation during the study period, the day of death or transplantation was considered end of study date for calculation of antibiotics and hospitalisation. These patients did not have a second PA antibody sample for obvious reasons.

All data were collected in a common database (Microsoft Access ${ }^{\circledR)}$ ) derived from the Danish CF Registry template with an additional project sheet added. Definitions for fields were common for all centers.

\section{Definition of chronic Pseudomonas aeruginosa infection:}

Chronic PA infection was defined as anti-Pseudomonas antibodies (serum precipitins) of $\geq 2$ and/or $\geq 3$ positive cultures over a six month period, and PA colonisation status was recorded both at 
inclusion and end of study ${ }^{19 ; 20}$. Further description on the method of antibody assays is reported in a separate publication ${ }^{21}$ Furthermore, chronic infection with Burkholderia species was recorded at study start only, according to the definitions used at each center - usually $\geq 3$ positive cultures over a six month period

\section{Statistics:}

Calculations were performed using SPSS version 14.0/15.0. Chi-square test was used for dichotomous variables (medication, infections), Mann-Whitney test was used for non-normally distributed variables (i.v. antibiotics, days of hospitalisation, $\mathrm{FEV}_{1}$, age at inclusion, age at diabetes), Student's t-test was used for normally distributed variables (BMI z-score). Linear regression analysis was used for overall analysis of FEV-1 and BMI vs gender, correcting for age, as well as i.v. antibiotics and gender corrected for chronic PA infection.

\section{$\underline{\text { Ethics }}$}

The study was approved by the regional ethical committees in the three countries, and informed consent was obtained from each participant and/or their guardian(-s).

Deleted: . II 


\section{RESULTS}

\section{Patients:}

Since we found that the prevalence of pancreatic sufficiency varied considerable between centers ( 0.4 to $26 \%$ ) and countries (1.9 to $16.9 \%)$ in the larger study (13) it was decided to include only the 890 CF patients (416 females) who were pancreatic insufficient for this part of the project.

We found a significantly higher number of males in total, primarily originating in a significantly excess of males in the adult subgroup (table 1).

The patients were studied for a mean of 374 days. Twelve females $(3 \%)$ and six males $(1 \%)$ died or had a lung transplantation during the study period ( $\mathrm{p}=0.087)$. Median age of death/ lung transplantation was 25.0 (range 10.7-50.1) years for females and 22.4 (range 10.2 - 29.1) years for males $(\mathrm{p}=0.25)$.

\section{Infection:}

At inclusion, 194 (46\%) females and 194 (41\%) males were chronically infected with PA $(\mathrm{p}=0.087)$. For patients $<19$ years the prevalence of chronic PA infection was $25.4 \%$ in females and $18.8 \%$ in males $(\mathrm{p}=0.07)$. In adults the prevalence was $78.0 \%$ in females and $69.8 \%$ in males $(\mathrm{p}=0.07)$. Females had a higher prevalence in all 5-year age-groups except $0-4 \mathrm{yr}$ olds, though only statistically significant in the $15-19$ yr and $30+$ groups (fig1). Of the 455 patients evaluated at the end of the study and not chronically infected at inclusion, 19/196 (9.7\%) females and 13/259 (5.0\%) males became chronically infected during the study period $(\mathrm{p}=0.053)$, with a significant difference in the adult population, $10 / 33(30.3 \%)$ vs $4 / 56(7.1 \%)$ respectively $(\mathrm{p}=0.004)$, but not in the pediatric group, $9 / 163(5.5 \%)$ vs. $9 / 203(4.4 \%)(\mathrm{p}=0.64)$.

Twenty-one females (5.0\%) and 11 males $(2.3 \%)$ were chronically infected with Burkholderia subspecies $(\mathrm{p}=0.029)$ at inclusion. 


\section{Clinical data and complications:}

We found no gender differences in lung function $\left(\mathrm{FEV}_{1} \%\right.$ of predicted) or BMI z-score, neither in all patients pooled, nor in any single age subgroup (table 1, fig 2). Neither did linear regression of FEV-1 and BMI z-score with correction for age show any difference either $(\mathrm{p}=0.69$ for FEV-1 and $\mathrm{p}=0.98$ for BMI).

Sixty females (14.4\%) were diagnosed with CFRD versus 59 males (12.4\%), $\mathrm{p}=0.39$. The females were diagnosed with CFRD at a younger age, but this difference was not significant (table 1).

\section{Treatment:}

During the follow up period, adult females received i.v. antibiotics of any kind in a median of 40 days ( $25 \%$ percentile 10 days; $75 \%$ percentile 54 days) per year versus 26 days $(0 ; 49)$ for males $(\mathrm{p}=0.009)$. No significant difference in use of i.v. antibiotics was found in the children (females median $0(0 ; 21)$ days, males median $0(0 ; 13)$ days; $\mathrm{p}=0.068)$. Correction for chronic PA infection by linear regression maintained a significantly higher use of i.v. antibiotics in adult females $(p=$ 0.027), but not for the children $(\mathrm{p}=0.538)$.

Median days/year on inhaled antibiotics was higher in adult females (27 days/year $(0 ; 293)$ vs. 0 days/year $(0 ; 302)$ in males), although far from significant $(\mathrm{p}=0.50)$. Correction for chronic PA infection by linear regression did not change this $(\mathrm{p}=0.90)$.

The adult females spent more days in hospital per year ( $\mathrm{p}=0.007$ ) (fig 3 ). We found no difference in the pediatric population (females median $0(0 ; 2.6)$ days vs males median $0(0 ; 0.90)$ days, $\mathrm{p}=$ $0.20)$.

The frequency of patients receiving inhaled or oral steroid was significantly higher among women (table 2). 
A total of 127 females (30.6\%) and 117 males (24.6\%) received continuous macrolide treatment (p $=0.048$ ). It was more common to prescribe macrolides to the female pediatric population both as a whole and looking at non-chronically infected patients separately, while there was no gender difference in the adult population (table 3). Looking at only the chronically infected patients, an equal number of the two genders received macrolides (females 101/218 (46.3\%) vs males 102/209 $(48.8 \%) ; \mathrm{p}=0.61)$, with no difference in either adults or children (table 3$)$.

Adult males received significantly more ursodeoxycholic acid (UDCA) than females (51/208 $(24.5 \%)$ vs. $25 / 168(14.9 \%) ; \mathrm{p}=0.021)$. Concerning the use of mucolytics (dornase alpha, $\mathrm{n}$ acetylcystein, bromhexin) no gender differences were observed (data not shown). 


\section{CONCLUSIONS}

The present study found no significant gender differences in key clinical factors such as lung

function or nutritional status in a Scandinavian CF cohort with PI. However, we found significantly more males than females in the adult population. Due to the design of this study, this does not prove $\underline{\text { a higher mortality in females as reported in several studies }} \stackrel{1: 3: 5}{v_{-}}$The difference in number of patients developing end stage lung disease (death or lung transplantation) in the one year follow-up

Deleted: the fact that there were more adult males compared to females is consistent with the higher mortality in females reported earlier

Deleted: Whether this reflects a higher mortality now or in the past cannot be answered by the design of this study. For further elucidation of present mortality a much longer prospective study is needed
muction period was not significant, although twice as many females died or were transplanted during the

period. However, this, as well as the surplus of males in the adult population hint that there may be a difference, which should be investigated in a much longer prospective study.

For the pediatric CF population in Scandinavia, the finding of no gender difference in key clinical

Deleted: However, $\mathrm{f}$ parameters is encouraging. This finding corresponds with the study by Verma et al indicating that modern intensive treatment may diminish gender difference in the CF population ${ }^{12}$.

The key novel finding in our study was that even without difference in lung function and BMI, we saw a distinct gender imbalance for some of the important complications and treatment modalities studied, indicating a persistent female downside.

The female patients tended to become chronically infected with PA earlier and more frequently than males, even though this was not significant except in smaller subgroups. The female incidence of new chronic infection was higher in the follow-up period among adult patients. The female patients also had significantly higher prevalence of chronic Burkholderia infection, an infection closely related to high morbidity and mortality ${ }^{22 ; 23}$. We found no significant difference in age or frequency at CFRD diagnosis, even if the median age of diabetic patients was three years lower in females. All of these factors predispose to a more severe course of the disease. 
Our study suggests that females may actually have a clinically more severe disease than males regardless of similar BMI and lung function.Some major differences in the treatment of male and

female patients were noticed,

Deleted: in this study

The females had a significantly higher use of i.v. antibiotics than the males (1.5 times higher median days per year) and spent more days in hospital regardless of differences in frequency of chronic PA infection. In Scandinavia, home i.v. treatment is widely used and accepted, indicating that primarily patients with severe symptoms are hospitalised. Consequently, the higher number of hospital days is not explained solely by the higher number of days with i.v. antibiotics, but imply that the females are in higher need for hospitalisation despite equal lung function.

The use of inhaled and oral steroids also differed which was somewhat unexpected. No gender difference in asthmatic symptoms in $\mathrm{CF}$ has been described. In non-CF asthma, however, a gender difference is reported, in that asthma is more prevalent in male children compared to females whereas it is more prevalent in adult women than in men ${ }^{24 ; 25}$. This difference is, however, not as high as the difference in use of steroids in this study (female odds ratio of two or more for both oral and inhaled steroid, table 2). The centres were not questioned about their policy for prescribing corticosteroids, especially whether a strict asthma diagnosis with positive reversibility was required. Hence, the difference may reflect a true difference in prevalence of asthma, or it may reflect a generally more severe lung disease in the females leading the clinicians to treat them more intensively, despite the lack of evidence of the efficacy of steroids in $\mathrm{CF}^{26 ; 27}$. Second generation macrolides, especially azithromycin, are used as low dose continuous treatment in $\mathrm{CF}$, especially in patients chronically infected with $\mathrm{PA}{ }^{15 ; 28}$. In the children $<19$ years, we found a higher use of macrolides in girls compared to boys. This could not be ascribed to an earlier and higher frequency of chronic pseudomonas infection, since the difference was still significant when excluding these patients. Actually more than $1 / 3$ of the children receiving continuous macrolide 
treatment had no chronic Gram negative infection. Whether these patients were patients at the verge of becoming chronically infected was not evident from the study design. This might indicate higher treatment intensity in female patients, also in the pediatric group.

As these data suggest, at least it seems that the female patients are treated more aggressively by their physicians. Whether this is due to a true difference in severity of disease or because the female

Deleted: Our study suggests that females may actually have a clinically more severe disease than males regardless of similar BMI and lung function.

Deleted: A patients present more symptoms to the physician leading him/her to intensify the treatment, is yet to be determined. Also the knowledge of a gender gap may lead the physicians to treat the female patients more aggressively.

Sex hormones are obvious candidates for causing the gender difference. A lack of the anabolic effect of testosterone in females is one of the proposed mechanisms, especially in CFRD ${ }^{5}$. This at least deserves more thorough investigation. An interesting recent work by Coakley et al, showing that estrogens may inhibit the alternative chloride transport, especially around ovulation when concentrations peak ${ }^{11}$, may explain one of the very basic differences between male and female $\mathrm{CF}$ patients. The reduced chloride transport will lead to an estimated monthly one-week period of more viscous mucus which may cause decreased lung function, increased risk of infection and need for antibiotics. This finding also correlates with our earlier study on lung function changes during the menstrual cycle ${ }^{10}$, and definitely warrants further investigation, both concerning anti-estrogen therapy as suggested by the authors, but also in the choice of anti-contraceptive therapy for female CF patients.

We found no significant difference concerning CFRD in our study, even though females tended to be diagnosed at an earlier age. According to previous studies, females seem to do worse after diagnosis of CFRD, but since such a gender difference is not seen in common IDDM, this does not explain the underlying difference, and may be due to the influence of CFRD on other aspects of CF, 
e.g. lung infections ${ }^{5}$. Milla et al did not account for pseudomonas status in their work. Thus, the possibility that poorer outcome is due to a higher prevalence of chronic infection does exist.

In conclusion, in a Scandinavian CF cohort from 2001-2003, we found no gender difference in key

Deleted: a deficit of female patients in the adult population, suggesting a higher clinical parameters such as FEV-1 and BMI in the included non-transplanted patients. We found a mortality for the females, but surplus of males in the adult population, but the design of this study does not allow any conclusion about differences in mortality. However, we did observe gender differences including higher risk of Pseudomonas and Burkholderia infection in females, and more intensified treatment in females regarding intravenous antibiotics, macrolides, steroids and hospitalization. Even if such intensified treatment can eventually even out the difference in mortality, there is still an obvious difference in morbidity and treatment burden, and the underlying reasons for this deserve further investigation. 


\section{ACKNOWLEDGEMENTS}

We thank all the CF-patients in each centre who have patiently participated in this study. We also thank the co-workers and the participators of the SCFSC for the effort and thoroughness during the work. Finally, we thank Solvay Pharma for the independent support which made the meetings of the SCFSC possible.

\section{FUNDING}

Solvay Pharma Denmark, Norway and Sweden funds the expenses at the consortium meetings with an unrestricted grant and with no influence on the scientific work of the consortium.

This study was supported partly by The Swedish Cystic Fibrosis Association.

\section{COMPETING INTERESTS}

None declared 


\section{FIGURE LEGENDS}

Fig 1:

Prevalence of chronic infection with Pseudomonas aeruginosa in different age groups. *= significant difference between male and females

Fig 2:

Lung function $\left(\mathrm{FEV}_{1} \%\right.$ of predicted) and BMI z-score according to gender in different 5-year age groups. Dark grey is male, light grey is female. Horizontal bars represent median, box represent interquartile range. No statistically significant difference in any age group.

Fig 3.

Days of hospitalisation in adult patients. Horizontal bars represent median, box represent interquartile range. Whiskers represent $95 \%$ percentiles. Dots and stars represent outliers and extreme outliers. $\mathrm{P}=0.007$. 
Reference List

(1) Kerem E, Reisman J, Corey M, Canny GJ, Levison H. Prediction of mortality in patients with cystic fibrosis. N Engl J Med 1992; 326(18):1187-1191.

(2) Konstan MW, Morgan WJ, Butler SM, Pasta DJ, Craib ML, Silva SJ et al. Risk factors for rate of decline in forced expiratory volume in one second in children and adolescents with cystic fibrosis. J Pediatr 2007; 151(2):134-9, 139.

(3) Rosenfeld M, Davis R, FitzSimmons S, Pepe M, Ramsey B. Gender gap in cystic fibrosis mortality. Am J Epidemiol 1997; 145(9):794-803.

(4) Marshall BC, Butler SM, Stoddard M, Moran AM, Liou TG, Morgan WJ. Epidemiology of cystic fibrosis-related diabetes. J Pediatr 2005; 146(5):681-687.

(5) Milla CE, Billings J, Moran A. Diabetes is associated with dramatically decreased survival in female but not male subjects with cystic fibrosis. Diabetes Care 2005; 28(9):2141-2144.

(6) Levy H, Kalish LA, Cannon CL, Garcia KC, Gerard C, Goldmann D et al. Predictors of mucoid Pseudomonas colonization in cystic fibrosis patients. Pediatr Pulmonol 2008; 43(5):463-471.

(7) Collins CE, O'Loughlin EV, Henry R. Discrepancies between males and females with cystic fibrosis in dietary intake and pancreatic enzyme use. J Pediatr Gastroenterol Nutr 1998; 26(3):258-262.

(8) Selvadurai HC, Blimkie CJ, Cooper PJ, Mellis CM, Van Asperen PP. Gender differences in habitual activity in children with cystic fibrosis. Arch Dis Child 2004; 89(10):928-933.

(9) Walters S. Sex differences in weight perception and nutritional behaviour in adults with cystic fibrosis. J Hum Nutr Diet 2001; 14(2):83-91.

(10) Johannesson M, Ludviksdottir D, Janson C. Lung function changes in relation to menstrual cycle in females with cystic fibrosis. Respir Med 2000; 94(11):1043-1046.

(11) Coakley RD. 17+i-Estradiol inhibits Ca2+-dependent homeostasis of airway surface liquid volume in human cystic fibrosis airway epithelia. J Clin Invest 2008; 118(12):4025-4035.

(12) Verma N, Bush A, Buchdahl R. Is there still a gender gap in cystic fibrosis? Chest 2005; 128(4):2824-2834.

(13) Knudsen PK, Olesen HV, Hoiby N, Johannesson M, Karpati F, Laerum BN et al. Differences in prevalence and treatment of Pseudomonas aeruginosa in cystic fibrosis centres in Denmark, Norway and Sweden. J Cyst Fibros 2009; 8(2):135-142.

(14) Word Health Organisation. Definition, Diagnosis and Classification of Diabetes Mellitus and its Complications; WHO/NCD/NCS/99.2 1999. http://whqlibdoc who int/hq/1999/WHO_NCD_NCS_99 2 pdf [ 1999 Available from: URL:http://whqlibdoc.who.int/hq/1999/WHO_NCD_NCS_99.2.pdf

(15) Wolter J, Seeney S, Bell S, Bowler S, Masel P, McCormack J. Effect of long term treatment with azithromycin on disease parameters in cystic fibrosis: a randomised trial. Thorax 2002; 57(3):212-216.

(16) Nysom K, Molgaard C, Hutchings B, Michaelsen KF. Body mass index of 0 to 45-y-old Danes: reference values and comparison with published European reference values. Int J Obes Relat Metab Disord 2001; 25(2):177184. 
(17) Quanjer PH, Tammeling Gj. Summary of recommendations. Standardized lung function testing. Report Working Party, European Community for Coal and Steel. Bull Eur Physiopathol Respir 1983; 19(S5):7-10.

(18) Solymar L, Aronsson PH, Bake B, Bjure J. Nitrogen single breath test, flow-volume curves and spirometry in healthy children, 7-18 years of age. Eur J Respir Dis 1980; 61(5):275-286.

(19) Hoiby N. Pseudomonas aeruginosa infection in cystic fibrosis. Diagnostic and prognostic significance of pseudomonas aeruginosa precipitins determined by means of crossed immunoelectrophoresis. A survey. Acta Pathol Microbiol Scand Suppl 1977;(262):1-96.

(20) Pedersen SS, Espersen F, Hoiby N. Diagnosis of chronic Pseudomonas aeruginosa infection in cystic fibrosis by enzyme-linked immunosorbent assay. J Clin Microbiol 1987; 25(10):1830-1836.

(21) Pressler T, Karpati F, Granstrom M, Knudsen PK, Lindblad A, Hjelte L et al. Diagnostic significance of measurements of specific IgG antibodies to Pseudomonas aeruginosa by three different serological methods. J Cyst Fibros 2009; 8(1):37-42.

(22) Govan JR, Deretic V. Microbial pathogenesis in cystic fibrosis: mucoid Pseudomonas aeruginosa and Burkholderia cepacia. Microbiol Rev 1996; 60(3):539-574.

(23) Ledson MJ, Gallagher MJ, Jackson M, Hart CA, Walshaw MJ. Outcome of Burkholderia cepacia colonisation in an adult cystic fibrosis centre. Thorax 2002; 57(2):142-145.

(24) Manfreda J, Sears MR, Becklake MR, Chan-Yeung M, mich-Ward H, Siersted HC et al. Geographic and gender variability in the prevalence of bronchial responsiveness in Canada. Chest 2004; 125(5):1657-1664.

(25) Sunyer J, Anto JM, Kogevinas M, Barcelo MA, Soriano JB, Tobias A et al. Risk factors for asthma in young adults. Spanish Group of the European Community Respiratory Health Survey. Eur Respir J 1997; 10(11):2490-2494.

(26) Balfour-Lynn IM, Lees B, Hall P, Phillips G, Khan M, Flather M et al. Multicenter randomized controlled trial of withdrawal of inhaled corticosteroids in cystic fibrosis. Am J Respir Crit Care Med 2006; 173(12):1356-1362.

(27) Cheng K, Ashby D, Smyth R. Oral steroids for cystic fibrosis. Cochrane Database Syst Rev 2000;(2):CD000407.

(28) Baumann U, Fischer JJ, Gudowius P, Lingner M, Herrmann S, Tummler B et al. Buccal adherence of Pseudomonas aeruginosa in patients with cystic fibrosis under long-term therapy with azithromycin. Infection 2001; 29(1):7-11. 


\section{GENDER DIFFERENCES IN THE SCANDINAVIAN CYSTIC}

\section{FIBROSIS POPULATION}

1. Hanne Vebert Olesen; Department of Paediatrics, Aarhus University Hospital Skejby, DK-8200 Aarhus N, Denmark

2. Tacjana Pressler; Cystic fibrosis Center, Department of Paediatrics, Copenhagen University Hospital, Copenhagen, Denmark

3. Lena Hjelte; Stockholm CF Center, Karolinska University Hospital Huddinge, Karolinska Institutet, SE-141 86 Stockholm, Sweden

4. Lena Mared; Lund CF Center, Heart and Lung Center, Lund University Hospital, SE-221 85 Lund, Sweden

5. Anders Lindblad; Gothenburg Cystic Fibrosis Center, Sahlgrenska University Hospital, SE-416 85 Gothenburg, Sweden

6. Per Kristian Knudsen; National Center for Cystic Fibrosis, Oslo University Hospital Ulleval, N0407 Oslo, Norway.

7. Birger N. Lærum; Department of Thoracic Medicine, Haukeland University Hospital, N-5021 Bergen, Norway

8. Marie Johannesson; Department of Public Health and Caring Sciences; Family Medicine and Clinical Epidemiology Section, Uppsala University, 751 05, Uppsala, Sweden, and Department of Paediatrics and Child Health, University of Otago, ,Wellington Campus, Wellington, New Zealand

On behalf of the Scandinavian Cystic Fibrosis Study Consortium* 
*Scandinavian Cystic Fibrosis Study Consortium (additional members):

Norway:

Pål Leyell Finstad, National Center for Cystic Fibrosis, Oslo University Hospital Ullevaal, Oslo;

Gjermund Fluge, Department of Pediatrics, Haukeland University Hospital, Bergen;

Helge Michalsen, Pediatric Department, Sørlandet Hospital, Arendal;

Bjørn Skrede, National Center for Cystic Fibrosis, Oslo University Hospital Ullevaal, Oslo;

Olav-Trond Storrøsten, National Center for Cystic Fibrosis, Oslo University Hospital Ulleval, Oslo.

Nils Olav Hermansen, Department of Microbiology, Oslo University Hospital Ullevaal, Oslo.

Sweden:

Anne Geborek, Stockholm Cystic Fibrosis Centre, Karolinska University Hospital Huddinge, Stockholm;

Marita Gilljam, Dept Pulmonary Medicine and Allergology, Sahlgrenska University Hospital, Gothenburg;

Ferenc Karpati, Stockholm Cystic Fibrosis Centre, Karolinska University Hospital Huddinge, Karolinska

Institutet, Stockholm;

Mary Kämpe, Uppsala CF Center, Uppsala University Hospital, Uppsala,

Peter Meyer, Heart and Lung Center, Lund University Hospital, Lund;

Denmark:

Peter Oluf Schiøtz, Department of Pediatrics, Aarhus University Hospital Skejby, Aarhus;

Niels Høiby, Department of Clinical Microbiology, Copenhagen University Hospital, Copenhagen

\author{
Corresponding author: Hanne Vebert Olesen, Aarhus University Hospital Skejby, \\ Department of Pediatrics, Brendstrupgaardsvej 100, 8200 Aarhus N, Denmark. \\ E-mail: hvo@dadlnet.dk; Phone: +45 894967 40; fax: +45 89496023
}


Part of this study was presented as an oral presentation at the $29^{\text {th }}$ European Cystic Fibrosis Conference, Copenhagen, June 15-18 2006 


\begin{abstract}
Aims.

To explore whether gender differences in the Scandinavian CF patients exist in the areas of key clinical parameters, complications and medication.
\end{abstract}

Methods. Cross-sectional data on 890 (416 female) pancreatic insufficient CF patients were evaluated regarding chronic infection, body mass index, lung function, medication, and diabetes, as well as data of pseudomonas infection status, antibiotic treatment and hospitalization from one year follow-up.

Results. We found no differences in lung function, body mass index, or frequency of diabetes. The adult group consisted of more males than females (208:168). We found no significant difference in prevalence of chronic Pseudomonas aeruginosa infection, but during the follow-up the incidence of new chronic infection was higher in adult females (10/33 vs. 4/56). Females had higher prevalence of Burkholderia infection (21/416 vs 11/474).

Adult females had more days on intravenous antibiotics (median 39 vs. 26 days/y), and days in hospital (median 2 vs 0 days/y). More adult females received inhaled and oral steroids. In the pediatric cohort, females were treated more often with macrolides as an anti-inflammatory agent.

Conclusion. We found no gender difference in key clinical parameters in our CF population.

However, our study showed a higher risk of Pseudomonas and Burkholderia infection among the female patients. Additionally, we found that female patients require more intensified treatment regarding antibiotics, macrolides, steroids and days of hospitalization, indicating a true female disadvantage even with modern aggressive treatment. 
The finding of more males than females in the adult population suggesting a male advantage, warrants a mortality study. 


\section{BACKGROUND}

A gender gap is documented in the course of cystic fibrosis (CF) with a female disadvantage regarding morbidity and mortality ${ }^{1-3}$. Several factors have been suggested as contributing to this gap. In CF related diabetes (CFRD), both earlier debut and considerably poorer survival in females have been reported ${ }^{4 ; 5}$. Chronic infection with Pseudomonas aeruginosa occurs earlier in females ${ }^{6}$. While these factors all contribute to morbidity and mortality, they do not explain the underlying difference. A few studies have tried to evaluate this difference. Focus has been on psychosocial differences, as well as hormonal differences. Female patients have less energy intake than males (compared to daily recommended intake), perform less physical exercise, and express an overestimation of their own weight ${ }^{7-9}$. Variations in lung function during the menstrual cycle points to effects of female sex hormones ${ }^{10}$, recently supported by a study of the influence of estrogen on the function of alternative chloride channels ${ }^{11}$. Other authors have suggested the lack of anabolic effects of male sex hormones as a factor ${ }^{5}$. Hence, there is considerable evidence that a true gender gap exists in cystic fibrosis. However, a recent single-center study showed no gender gap when comparing key values like lung function, height and weight in children and adolescents ${ }^{12}$. The authors suggest that a closing of the gap is possible, even mandatory, with standardized intensive care.

\section{Working hypothesis:}

No gender differences exist among Scandinavian Cystic Fibrosis patients since by tradition we have a very proactive and easily accessible care for all our patients.

\section{Aims:}

To explore whether gender differences in the Scandinavian CF patients exist in the areas of key clinical parameters, complications and medication. 


\section{METHODS:}

\section{Study design:}

This study was part of a larger multi centre study on the prevalence and incidence of Pseudomonas aeruginosa $(\mathrm{PA})$ infection in the Scandinavian $\mathrm{CF}$ cohort ${ }^{13}$. All eight CF centres in Scandinavia participated. Data were collected from 2001 to 2003. Overall, all Scandinavian countries have very high standards of living, and a government-financed health system with a high standard of centralised CF care. Some differences in CF care do exist however, such as a higher degree of shared care in northern Sweden and Norway due to geography, and somewhat different antibiotic and segregation policies. The differences between countries and centres have been presented in the main article within this project ${ }^{13}$. For the scope of this article, we focused only on the gender perspective. The treatment policies for male and female CF patients were similar within the individual center.

All CF patients, followed at the eight Scandinavian CF centres at the time of inclusion, were eligible for the study. Approximately $86 \%$ of all patients participated, allowing for a $>90 \%$ participation of patients eligible for the study (= non-transplanted). Patients were recruited consecutively at routine visits to the centres, when in a clinically stable condition. Exclusion criterion was lung transplant prior to inclusion, since this was likely to change the infection status, lung function and body mass index of the patients. CF was diagnosed as either two known CFcausing mutations in the CF transmembrane conductance regulator (CFTR) gene, and/or two positive sweat-tests together with symptoms compatible with the disease. Data recorded at inclusion were: age, height, weight, BMI, lung function $\left(\mathrm{FEV}_{1}\right)$, chronic infections (based on regular cultures, 
as well as antibodies against PA, see below), regular medication at time of inclusion, pancreatic insufficiency (PI), CF related diabetes (CFRD) and meconium ileus (MI). Patients using pancreatic enzymes were classified as PI. Diabetes was defined as CFRD with fasting hyperglycemia using the WHO criteria ${ }^{14}$. Continuous macrolide treatment was recorded exclusively for patients with this drug prescribed as an anti-inflammatory agent ${ }^{15}$. Other regular medication was recorded if prescribed at inclusion (oral and inhaled steroids, ursodeoxycholic acid, mucolytics, and insulin). The patients were followed for one year, recording number of days in hospital and number of days on inhaled or intravenous (i.v.) antibiotics. At the end of the study period new PA antibodies were measured and infection status was reported. Patients 19 years or older at inclusion were designated as adults. For calculation of BMI z-score the reference values of Nysom was used ${ }^{16}$. For patients $\geq 19$ years the reference values from European Coal and Steel Community were used for calculating $\mathrm{FEV}_{1}$ percent of predicted $^{17}$, and for those $<19$ years Solymar reference values were used ${ }^{18}$. Days on inhaled or intravenous antibiotics and hospitalisation during the study period were recorded and calculated as days/year. For patients who died or underwent lung transplantation during the study period, the day of death or transplantation was considered end of study date for calculation of antibiotics and hospitalisation. These patients did not have a second PA antibody sample for obvious reasons.

All data were collected in a common database (Microsoft Access ${ }^{\circledR}$ ) derived from the Danish CF Registry template with an additional project sheet added. Definitions for fields were common for all centers.

\section{Definition of chronic Pseudomonas aeruginosa infection:}

Chronic PA infection was defined as anti-Pseudomonas antibodies (serum precipitins) of $\geq 2$ and/or $\geq 3$ positive cultures over a six month period, and PA colonisation status was recorded both at 
inclusion and end of study ${ }^{19 ; 20}$. Further description on the method of antibody assays is reported in a separate publication ${ }^{21}$ Furthermore, chronic infection with Burkholderia species was recorded at study start only, according to the definitions used at each center - usually $\geq 3$ positive cultures over a six month period

\section{Statistics:}

Calculations were performed using SPSS version 14.0/15.0. Chi-square test was used for dichotomous variables (medication, infections), Mann-Whitney test was used for non-normally distributed variables (i.v. antibiotics, days of hospitalisation, $\mathrm{FEV}_{1}$, age at inclusion, age at diabetes), Student's t-test was used for normally distributed variables (BMI z-score). Linear regression analysis was used for overall analysis of FEV-1 and BMI vs gender, correcting for age, as well as i.v. antibiotics and gender corrected for chronic PA infection.

\section{Ethics}

The study was approved by the regional ethical committees in the three countries, and informed consent was obtained from each participant and/or their guardian(-s). 


\section{RESULTS}

\section{Patients:}

Since we found that the prevalence of pancreatic sufficiency varied considerable between centers (0.4 to $26 \%)$ and countries (1.9 to $16.9 \%)$ in the larger study (13) it was decided to include only the $890 \mathrm{CF}$ patients (416 females) who were pancreatic insufficient for this part of the project.

We found a significantly higher number of males in total, primarily originating in a significantly excess of males in the adult subgroup (table 1).

The patients were studied for a mean of 374 days. Twelve females (3\%) and six males (1\%) died or had a lung transplantation during the study period $(\mathrm{p}=0.087)$. Median age of death/ lung transplantation was 25.0 (range 10.7-50.1) years for females and 22.4 (range 10.2 - 29.1) years for males $(\mathrm{p}=0.25)$.

\section{Infection:}

At inclusion, 194 (46\%) females and 194 (41\%) males were chronically infected with PA $(\mathrm{p}=0.087)$. For patients $<19$ years the prevalence of chronic PA infection was $25.4 \%$ in females and $18.8 \%$ in males $(\mathrm{p}=0.07)$. In adults the prevalence was $78.0 \%$ in females and $69.8 \%$ in males $(\mathrm{p}=0.07)$. Females had a higher prevalence in all 5-year age-groups except $0-4$ yr olds, though only statistically significant in the $15-19 \mathrm{yr}$ and $30+$ groups (fig1). Of the 455 patients evaluated at the end of the study and not chronically infected at inclusion, 19/196 (9.7\%) females and 13/259 (5.0\%) males became chronically infected during the study period $(\mathrm{p}=0.053)$, with a significant difference in the adult population, $10 / 33(30.3 \%)$ vs $4 / 56(7.1 \%)$ respectively $(p=0.004)$, but not in the pediatric group, $9 / 163(5.5 \%)$ vs. 9/203 $(4.4 \%)(\mathrm{p}=0.64)$.

Twenty-one females (5.0\%) and 11 males $(2.3 \%)$ were chronically infected with Burkholderia subspecies $(\mathrm{p}=0.029)$ at inclusion. 


\section{Clinical data and complications:}

We found no gender differences in lung function ( $\mathrm{FEV}_{1} \%$ of predicted) or BMI z-score, neither in all patients pooled, nor in any single age subgroup (table 1, fig 2). Neither did linear regression of FEV-1 and BMI z-score with correction for age show any difference either ( $p=0.69$ for FEV-1 and $\mathrm{p}=0.98$ for BMI).

Sixty females $(14.4 \%)$ were diagnosed with CFRD versus 59 males $(12.4 \%), \mathrm{p}=0.39$. The females were diagnosed with CFRD at a younger age, but this difference was not significant (table 1).

\section{Treatment:}

During the follow up period, adult females received i.v. antibiotics of any kind in a median of 40 days (25\% percentile 10 days; $75 \%$ percentile 54 days) per year versus 26 days $(0 ; 49)$ for males $(\mathrm{p}=0.009)$. No significant difference in use of i.v. antibiotics was found in the children (females median $0(0 ; 21)$ days, males median $0(0 ; 13)$ days; $\mathrm{p}=0.068)$. Correction for chronic PA infection by linear regression maintained a significantly higher use of i.v. antibiotics in adult females ( $\mathrm{p}=$ 0.027), but not for the children $(\mathrm{p}=0.538)$.

Median days/year on inhaled antibiotics was higher in adult females (27 days/year (0;293) vs. 0 days/year $(0 ; 302)$ in males $)$, although far from significant $(\mathrm{p}=0.50)$. Correction for chronic PA infection by linear regression did not change this $(\mathrm{p}=0.90)$.

The adult females spent more days in hospital per year $(\mathrm{p}=0.007$ ) (fig 3 ). We found no difference in the pediatric population (females median $0(0 ; 2.6)$ days vs males median $0(0 ; 0.90)$ days, $\mathrm{p}=$ $0.20)$.

The frequency of patients receiving inhaled or oral steroid was significantly higher among women (table 2). 
A total of 127 females (30.6\%) and 117 males (24.6\%) received continuous macrolide treatment (p $=0.048)$. It was more common to prescribe macrolides to the female pediatric population both as a whole and looking at non-chronically infected patients separately, while there was no gender difference in the adult population (table 3). Looking at only the chronically infected patients, an equal number of the two genders received macrolides (females 101/218 (46.3\%) vs males 102/209 $(48.8 \%) ; \mathrm{p}=0.61)$, with no difference in either adults or children (table 3 ).

Adult males received significantly more ursodeoxycholic acid (UDCA) than females (51/208 $(24.5 \%)$ vs. $25 / 168(14.9 \%) ; p=0.021)$. Concerning the use of mucolytics (dornase alpha, $n-$ acetylcystein, bromhexin) no gender differences were observed (data not shown). 


\section{CONCLUSIONS}

The present study found no significant gender differences in key clinical factors such as lung function or nutritional status in a Scandinavian CF cohort with PI. However, we found significantly more males than females in the adult population. Due to the design of this study, this does not prove a higher mortality in females as reported in several studies ${ }^{1 ; 3 ; 5} .$. The difference in number of patients developing end stage lung disease (death or lung transplantation) in the one year follow-up period was not significant, although twice as many females died or were transplanted during the period. However, this, as well as the surplus of males in the adult population hint that there may be a difference, which should be investigated in a much longer prospective study.

For the pediatric CF population in Scandinavia, the finding of no gender difference in key clinical parameters is encouraging. This finding corresponds with the study by Verma et al indicating that modern intensive treatment may diminish gender difference in the CF population ${ }^{12}$. The key novel finding in our study was that even without difference in lung function and BMI, we saw a distinct gender imbalance for some of the important complications and treatment modalities studied, indicating a persistent female downside.

The female patients tended to become chronically infected with PA earlier and more frequently than males, even though this was not significant except in smaller subgroups. The female incidence of new chronic infection was higher in the follow-up period among adult patients. The female patients also had significantly higher prevalence of chronic Burkholderia infection, an infection closely related to high morbidity and mortality ${ }^{22 ; 23}$. We found no significant difference in age or frequency at CFRD diagnosis, even if the median age of diabetic patients was three years lower in females. All of these factors predispose to a more severe course of the disease. 
Our study suggests that females may actually have a clinically more severe disease than males regardless of similar BMI and lung function.Some major differences in the treatment of male and female patients were noticed.

The females had a significantly higher use of i.v. antibiotics than the males (1.5 times higher median days per year) and spent more days in hospital regardless of differences in frequency of chronic PA infection. In Scandinavia, home i.v. treatment is widely used and accepted, indicating that primarily patients with severe symptoms are hospitalised. Consequently, the higher number of hospital days is not explained solely by the higher number of days with i.v. antibiotics, but imply that the females are in higher need for hospitalisation despite equal lung function.

The use of inhaled and oral steroids also differed which was somewhat unexpected. No gender difference in asthmatic symptoms in CF has been described. In non-CF asthma, however, a gender difference is reported, in that asthma is more prevalent in male children compared to females whereas it is more prevalent in adult women than in men ${ }^{24 ; 25}$. This difference is, however, not as high as the difference in use of steroids in this study (female odds ratio of two or more for both oral and inhaled steroid, table 2). The centres were not questioned about their policy for prescribing corticosteroids, especially whether a strict asthma diagnosis with positive reversibility was required. Hence, the difference may reflect a true difference in prevalence of asthma, or it may reflect a generally more severe lung disease in the females leading the clinicians to treat them more intensively, despite the lack of evidence of the efficacy of steroids in $\mathrm{CF}^{26 ; 27}$.

Second generation macrolides, especially azithromycin, are used as low dose continuous treatment in $\mathrm{CF}$, especially in patients chronically infected with $\mathrm{PA}^{15 ; 28}$. In the children $<19$ years, we found a higher use of macrolides in girls compared to boys. This could not be ascribed to an earlier and higher frequency of chronic pseudomonas infection, since the difference was still significant when excluding these patients. Actually more than $1 / 3$ of the children receiving continuous macrolide 
treatment had no chronic Gram negative infection. Whether these patients were patients at the verge of becoming chronically infected was not evident from the study design. This might indicate higher treatment intensity in female patients, also in the pediatric group.

As these data suggest, at least it seems that the female patients are treated more aggressively by their physicians. Whether this is due to a true difference in severity of disease or because the female patients present more symptoms to the physician leading him/her to intensify the treatment, is yet to be determined. Also the knowledge of a gender gap may lead the physicians to treat the female patients more aggressively.

Sex hormones are obvious candidates for causing the gender difference. A lack of the anabolic effect of testosterone in females is one of the proposed mechanisms, especially in CFRD ${ }^{5}$. This at least deserves more thorough investigation. An interesting recent work by Coakley et al, showing that estrogens may inhibit the alternative chloride transport, especially around ovulation when concentrations peak ${ }^{11}$, may explain one of the very basic differences between male and female CF patients. The reduced chloride transport will lead to an estimated monthly one-week period of more viscous mucus which may cause decreased lung function, increased risk of infection and need for antibiotics. This finding also correlates with our earlier study on lung function changes during the menstrual cycle ${ }^{10}$, and definitely warrants further investigation, both concerning anti-estrogen therapy as suggested by the authors, but also in the choice of anti-contraceptive therapy for female CF patients.

We found no significant difference concerning CFRD in our study, even though females tended to be diagnosed at an earlier age. According to previous studies, females seem to do worse after diagnosis of CFRD, but since such a gender difference is not seen in common IDDM, this does not explain the underlying difference, and may be due to the influence of CFRD on other aspects of CF, 
e.g. lung infections ${ }^{5}$. Milla et al did not account for pseudomonas status in their work. Thus, the possibility that poorer outcome is due to a higher prevalence of chronic infection does exist.

In conclusion, in a Scandinavian CF cohort from 2001-2003, we found no gender difference in key clinical parameters such as FEV-1 and BMI in the included non-transplanted patients. We found a surplus of males in the adult population, but the design of this study does not allow any conclusion about differences in mortality. . However, we did observe gender differences including higher risk of Pseudomonas and Burkholderia infection in females, and more intensified treatment in females regarding intravenous antibiotics, macrolides, steroids and hospitalization. Even if such intensified treatment can eventually even out the difference in mortality, there is still an obvious difference in morbidity and treatment burden, and the underlying reasons for this deserve further investigation. 


\section{ACKNOWLEDGEMENTS}

We thank all the CF-patients in each centre who have patiently participated in this study. We also thank the co-workers and the participators of the SCFSC for the effort and thoroughness during the work. Finally, we thank Solvay Pharma for the independent support which made the meetings of the SCFSC possible.

\section{FUNDING}

Solvay Pharma Denmark, Norway and Sweden funds the expenses at the consortium meetings with an unrestricted grant and with no influence on the scientific work of the consortium.

This study was supported partly by The Swedish Cystic Fibrosis Association.

\section{COMPETING INTERESTS}

None declared 


\section{FIGURE LEGENDS}

Fig 1:

Prevalence of chronic infection with Pseudomonas aeruginosa in different age groups. *= significant difference between male and females

Fig 2:

Lung function ( $\mathrm{FEV}_{1} \%$ of predicted) and $\mathrm{BMI} z$-score according to gender in different 5-year age groups. Dark grey is male, light grey is female. Horizontal bars represent median, box represent interquartile range. No statistically significant difference in any age group.

Fig 3.

Days of hospitalisation in adult patients. Horizontal bars represent median, box represent interquartile range. Whiskers represent $95 \%$ percentiles. Dots and stars represent outliers and extreme outliers. $\mathrm{P}=0.007$. 


\section{Reference List}

(1) Kerem E, Reisman J, Corey M, Canny GJ, Levison H. Prediction of mortality in patients with cystic fibrosis. N Engl J Med 1992; 326(18):1187-1191.

(2) Konstan MW, Morgan WJ, Butler SM, Pasta DJ, Craib ML, Silva SJ et al. Risk factors for rate of decline in forced expiratory volume in one second in children and adolescents with cystic fibrosis. J Pediatr 2007; 151(2):134-9, 139.

(3) Rosenfeld M, Davis R, FitzSimmons S, Pepe M, Ramsey B. Gender gap in cystic fibrosis mortality. Am J Epidemiol 1997; 145(9):794-803.

(4) Marshall BC, Butler SM, Stoddard M, Moran AM, Liou TG, Morgan WJ. Epidemiology of cystic fibrosis-related diabetes. J Pediatr 2005; 146(5):681-687.

(5) Milla CE, Billings J, Moran A. Diabetes is associated with dramatically decreased survival in female but not male subjects with cystic fibrosis. Diabetes Care 2005; 28(9):2141-2144.

(6) Levy H, Kalish LA, Cannon CL, Garcia KC, Gerard C, Goldmann D et al. Predictors of mucoid Pseudomonas colonization in cystic fibrosis patients. Pediatr Pulmonol 2008; 43(5):463-471.

(7) Collins CE, O'Loughlin EV, Henry R. Discrepancies between males and females with cystic fibrosis in dietary intake and pancreatic enzyme use. J Pediatr Gastroenterol Nutr 1998; 26(3):258-262.

(8) Selvadurai HC, Blimkie CJ, Cooper PJ, Mellis CM, Van Asperen PP. Gender differences in habitual activity in children with cystic fibrosis. Arch Dis Child 2004; 89(10):928-933.

(9) Walters S. Sex differences in weight perception and nutritional behaviour in adults with cystic fibrosis. J Hum Nutr Diet 2001; 14(2):83-91.

(10) Johannesson M, Ludviksdottir D, Janson C. Lung function changes in relation to menstrual cycle in females with cystic fibrosis. Respir Med 2000; 94(11):1043-1046.

(11) Coakley RD. 17+i-Estradiol inhibits Ca2+-dependent homeostasis of airway surface liquid volume in human cystic fibrosis airway epithelia. J Clin Invest 2008; 118(12):4025-4035.

(12) Verma N, Bush A, Buchdahl R. Is there still a gender gap in cystic fibrosis? Chest 2005; 128(4):2824-2834.

(13) Knudsen PK, Olesen HV, Hoiby N, Johannesson M, Karpati F, Laerum BN et al. Differences in prevalence and treatment of Pseudomonas aeruginosa in cystic fibrosis centres in Denmark, Norway and Sweden. J Cyst Fibros 2009; 8(2):135-142.

(14) Word Health Organisation. Definition, Diagnosis and Classification of Diabetes Mellitus and its Complications; WHO/NCD/NCS/99.2 1999. http://whqlibdoc who int/hq/1999/WHO_NCD_NCS_99 2 pdf [ 1999 Available from: URL:http://whqlibdoc.who.int/hq/1999/WHO_NCD_NCS_99.2.pdf

(15) Wolter J, Seeney S, Bell S, Bowler S, Masel P, McCormack J. Effect of long term treatment with azithromycin on disease parameters in cystic fibrosis: a randomised trial. Thorax 2002; 57(3):212-216.

(16) Nysom K, Molgaard C, Hutchings B, Michaelsen KF. Body mass index of 0 to 45-y-old Danes: reference values and comparison with published European reference values. Int J Obes Relat Metab Disord 2001; 25(2):177184. 
(17) Quanjer PH, Tammeling Gj. Summary of recommendations. Standardized lung function testing. Report Working Party, European Community for Coal and Steel. Bull Eur Physiopathol Respir 1983; 19(S5):7-10.

(18) Solymar L, Aronsson PH, Bake B, Bjure J. Nitrogen single breath test, flow-volume curves and spirometry in healthy children, 7-18 years of age. Eur J Respir Dis 1980; 61(5):275-286.

(19) Hoiby N. Pseudomonas aeruginosa infection in cystic fibrosis. Diagnostic and prognostic significance of pseudomonas aeruginosa precipitins determined by means of crossed immunoelectrophoresis. A survey. Acta Pathol Microbiol Scand Suppl 1977;(262):1-96.

(20) Pedersen SS, Espersen F, Hoiby N. Diagnosis of chronic Pseudomonas aeruginosa infection in cystic fibrosis by enzyme-linked immunosorbent assay. J Clin Microbiol 1987; 25(10):1830-1836.

(21) Pressler T, Karpati F, Granstrom M, Knudsen PK, Lindblad A, Hjelte L et al. Diagnostic significance of measurements of specific IgG antibodies to Pseudomonas aeruginosa by three different serological methods. J Cyst Fibros 2009; 8(1):37-42.

(22) Govan JR, Deretic V. Microbial pathogenesis in cystic fibrosis: mucoid Pseudomonas aeruginosa and Burkholderia cepacia. Microbiol Rev 1996; 60(3):539-574.

(23) Ledson MJ, Gallagher MJ, Jackson M, Hart CA, Walshaw MJ. Outcome of Burkholderia cepacia colonisation in an adult cystic fibrosis centre. Thorax 2002; 57(2):142-145.

(24) Manfreda J, Sears MR, Becklake MR, Chan-Yeung M, mich-Ward H, Siersted HC et al. Geographic and gender variability in the prevalence of bronchial responsiveness in Canada. Chest 2004; 125(5):1657-1664.

(25) Sunyer J, Anto JM, Kogevinas M, Barcelo MA, Soriano JB, Tobias A et al. Risk factors for asthma in young adults. Spanish Group of the European Community Respiratory Health Survey. Eur Respir J 1997; 10(11):2490-2494.

(26) Balfour-Lynn IM, Lees B, Hall P, Phillips G, Khan M, Flather M et al. Multicenter randomized controlled trial of withdrawal of inhaled corticosteroids in cystic fibrosis. Am J Respir Crit Care Med 2006; 173(12):1356-1362.

(27) Cheng K, Ashby D, Smyth R. Oral steroids for cystic fibrosis. Cochrane Database Syst Rev 2000;(2):CD000407.

(28) Baumann U, Fischer JJ, Gudowius P, Lingner M, Herrmann S, Tummler B et al. Buccal adherence of Pseudomonas aeruginosa in patients with cystic fibrosis under long-term therapy with azithromycin. Infection 2001; 29(1):7-11. 
Prevalence of chronic infection with Pseudomonas aeruginosa in different age groups. $*=$ significant difference between male and females $254 \times 190 \mathrm{~mm}(96 \times 96 \mathrm{DPI})$

John Wiley \& Sons, Inc. 


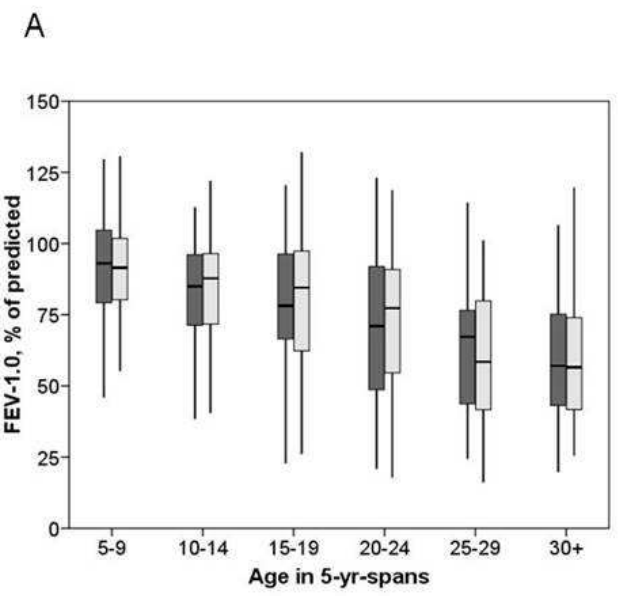

\section{B}



Lung function (FEV1\% of predicted) and BMI z-score according to gender in different 5-year age groups. Dark grey is male, light grey is female. Horizontal bars represent median, box represent interquartile range. No statistically significant difference in any age group.

$254 \times 190 \mathrm{~mm}(96 \times 96 \mathrm{DPI})$

John Wiley \& Sons, Inc. 
Days of hospitalisation in adult patients. Horizontal bars represent median, box represent interquartile range. Dots and stars represent outliers. $\mathrm{P}=0.007$. $254 \times 190 \mathrm{~mm}(96 \times 96$ DPI) 
Table 1. Gender differences in demograpic and clinical markers

\begin{tabular}{|c|c|c|c|c|}
\hline & & Female & Male & p-value \\
\hline $\begin{array}{l}\text { Number of patients } \\
(\%)\end{array}$ & $\begin{array}{l}\text { Total } \\
\text { Child } \\
\text { Adult }\end{array}$ & $\begin{array}{l}416(46.7) \\
248(48.2) \\
168(44.7)\end{array}$ & $\begin{array}{l}\mathbf{4 7 4}(\mathbf{5 3 . 3 )} \\
266(51.8) \\
208(55.3)\end{array}$ & $\begin{array}{l}\mathbf{0 . 0 5 2} \\
0.427 \S \\
0.039 \S\end{array}$ \\
\hline $\begin{array}{l}\text { Age in yrs at } \\
\text { inclusion (median, } \\
\text { (25;75 percentile), }\end{array}$ & $\begin{array}{l}\text { Total } \\
\text { Child } \\
\text { Adult }\end{array}$ & $\begin{array}{l}\mathbf{1 5 . 4}(\mathbf{8 . 7} ; 25.3) \\
10.2(0.4-18.9) \\
27.9(19-58.5)\end{array}$ & $\begin{array}{l}\mathbf{1 7 . 1}(\mathbf{9 . 0 ; 2 6 . 4 )} \\
9.7(0.3-18.8) \\
27.5(19-55.5)\end{array}$ & $\begin{array}{l}\mathbf{0 . 4 3} \\
0.91 \\
0.79\end{array}$ \\
\hline $\begin{array}{l}\text { BMI z-score (mean, } \\
\text { SD) }\end{array}$ & $\begin{array}{l}\text { Total } \\
\text { Child } \\
\text { Adult }\end{array}$ & $\begin{array}{l}\mathbf{- 0 . 2 8}(\mathbf{1 . 3 5}) \\
-0.07(1.41) \\
-0.59(1.20)\end{array}$ & $\begin{array}{l}\mathbf{- 0 . 2 9}(\mathbf{1 . 2 1}) \\
-0.06(1.07) \\
-0.59(1.31)\end{array}$ & $\begin{array}{l}\mathbf{0 . 9 1} \\
0.93 \\
0.96\end{array}$ \\
\hline $\begin{array}{l}\text { FEV }-1 \% \text { of } \\
\text { predicted (median } \\
(25 ; 75 \text { percentile) })\end{array}$ & $\begin{array}{l}\text { Total } \\
\text { Child } \\
\text { Adult }\end{array}$ & $\begin{array}{l}78(\mathbf{5 7 . 1 ; 9 5 . 0}) \\
87(16-131) \\
63(20-123)\end{array}$ & $\begin{array}{l}80(\mathbf{5 7 . 2 ; 9 4 . 2}) \\
88(26-135) \\
67(16-148)\end{array}$ & $\begin{array}{l}\mathbf{0 . 9 0} \\
0.85 \\
0.70\end{array}$ \\
\hline $\begin{array}{l}\text { Age in yrs at } \\
\text { diagnosis of CFRD\# } \\
\text { (median }(25 ; 75 \\
\text { percentile)) }\end{array}$ & Total & $16.8(14.0 ; 25.0)$ & $19.8(16.0 ; 29.3)$ & 0.083 \\
\hline
\end{tabular}


Table 2. Differences in use of steroids in adult female and male Scandinavian cystic fibrosis patients

\section{Female Male}

Steroids

$$
(\mathrm{n}=168) \quad(\mathrm{n}=208)
$$

p-value* $\quad$ OR $\quad 95 \%$ CI of OR

$\begin{array}{llllll}\text { Oral, } \mathrm{n}(\%) & 17(10.1) & 10(4.8) & \mathrm{p}=0.047 & 2.3 & 0.99-4.99 \\ & & & & & \\ \text { Inhaled, } \mathrm{n}(\%) & 73(43.5) & 58(27.9) & \mathrm{p}=0.002 & 2.0 & 1.30-3.00\end{array}$

*chi square; $\mathrm{OR}=$ odds ratio; $\mathrm{CI}=$ confidence interval 
Table 3. Gender differences in use of continuous macrolides in Scandinavian cystic fibrosis patients according to infection status.

Macrolide

No chronic infection

Chronic Gram negative infection antibiotics

$\begin{array}{lllllll} & \text { Female } & \text { Male } & \text { p-value* } & \text { Female } & \text { Male } & \text { p-value* } \\ \text { Children** } & 23(13.1 \%) & 14(6.7 \%) & \mathrm{p}=0.036 & 31(43.1 \%) & 24(41.4 \%) & \mathrm{p}=0.85 \\ \text { Adults*** } & 2(6.7 \%) & 2(4.2 \%) & \mathrm{p}=0.63 & 71(51.8 \%) & 77(48.1 \%) & \mathrm{p}=0.52\end{array}$

*chi square

** all child patients $54(22 \%) \mathrm{F}$ vs $38(14 \%) \mathrm{M} ; \mathrm{p}=0.027$

*** all adult patients $73(44 \%) \mathrm{F}$ vs $79(38 \%) \mathrm{M} ; \mathrm{p}=0.26$ 